\title{
Optimal Control of Decoupling Point with Deteriorating Items
}

\author{
Kuan Yang, Ermei Wang \\ School of Business Administration, Hunan University (China)
}

yangkuan@bnu.edu.cn,wemtb2009@163.com

Received: July 2014

Accepted: November 2014

\section{Abstract:}

Purpose: The aim of this paper is to develop a dynamic model to simultaneously determine the optimal position of the decoupling point and the optimal path of the production rate as well as the inventory level in a supply chain. With the objective to minimize the total cost of the deviation from the target setting, the closed forms of the optimal solution are derived over a finite planning horizon with deterioration rate under time-varying demand rate.

Design/methodology/approach: The Pontryagin's Maximum Principle is employed to explore the optimal position of decoupling point and the optimal production and inventory rate for the proposed dynamic models. The performances of parameters are illustrated through analytical and numerical approaches.

Findings: The results denote that the optimal production rate and inventory level are closely related to the target setting which are highly dependent on production policy; meanwhile the optimal decoupling point is exist and unique with the fluctuating of deteriorating rate and product life cycle. The further analyses through both mathematic and numerical approaches indicate that the shorten of product life cycle shifts the optimal decoupling point forward to the end customer meanwhile a backward shifting appears when the deterioration rate increase.

Research limitations/implications: There is no shortage allowed and the replacement policy is not taken into account. 
Practical implications: Solutions derived from this study of the optimal production-inventory plan and decoupling point are instructive for operation decision making. The obtained knowledge about the performance of different parameters is critical to deteriorating supply chains management.

Originality/value: Many previous models of the production-inventory problem are only focused on the cost. The paper introduces the decoupling point control into the production and inventory problem such that a critical element-customer demand, can be taken into account. And the problem is solved as dynamic when the production rate, inventory level and the position of the decoupling point are all regarded as decision variables.

Keywords: decoupling points, production-inventory management, optimal control theory, deteriorating items, time-varying demand, supply chain management

\section{Introduction}

With the emergence of a business era that embraces "change" as one of its major characteristics (Agarwal, Shankar \& Tiwari, 2006), the difficulty of success is increasing for many manufactures to a large extent. Since the customer needs and preferences deeply influenced the SC's inner workings such as product functionality, quality, speed of production, timeliness of deliveries and so on, manufacturers are no longer the sole drivers of the supply chain. A shift from a "push" to a "pull" environment is well on its way. Hence, there confront a very practical problem: how to take the customer demand into consideration while designing an enterprise' inner works such as production planning and inventory policy, and how to schedule these operational decisions in adjusting to demand changes.

A decoupling point is a boundary between "push" and "pull" to separates the order-driven activities from the forecast-driven activities. In terms of the delivery process, the "push" and "pull" is also equivalent to make-to-stock (MTS) and make-to-order (MTO) strategy respectively. From the upstream of the supply chain to the decoupling point the schedule is planned based on the forecast of market demands while the operations are driven by customer orders rather than prediction from the decoupling point to the down-stream of supply chain. In other words, the position of decoupling point indicates how deeply the customer order penetrates into the goods flow (Van Donk, 2001). As shown in Figure 1, between the MTS and MTO, the feasible positions of the decoupling point exist on any stage of the supply chain. 


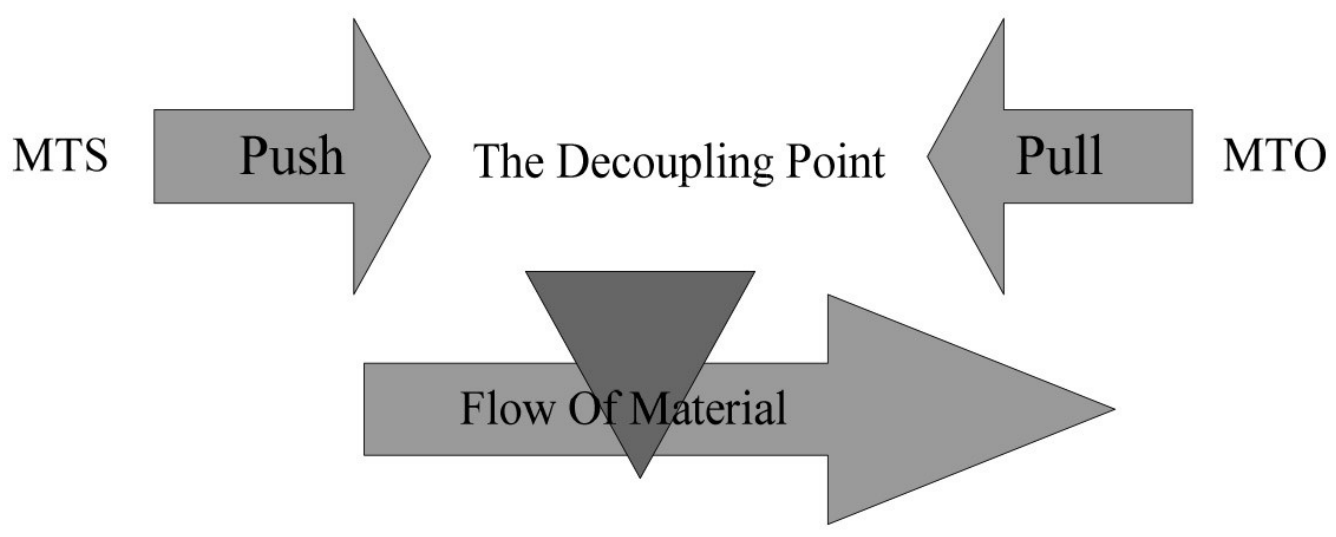

Figure 1. feasible positions of a decoupling point

In reality, there are many reasons and effects of shifting the decoupling point forward or backward to the end customers (Olhager, 2003). Soman, Van and Gaalman (2004) pointed out that the decoupling point decision is closely related to the production planning, inventory policy and operational decisions. The backward shifting away from the customer can raise product variety and customization with lower inventory risks, and the manufacturing efficiency improves but generally displaying longer customer lead times; meanwhile the forward shifting will fill customer orders quickly, whereas reduce the product customization (Jeong, 2011). It was obvious that the advantages of the backward shifting are the disadvantages of the forward shifting of the decoupling point and vice versa.

There exist two different approaches in determining the position of the decoupling point, conceptual model approaches and mathematical model approaches. The conceptual model approaches intend to provide guidelines using knowledge-based systems or case study for decoupling point selecting. To provide an efficacious approach in designing decoupling point, Hoekstra, Romme and Argelo (1992) furnish a concept that the critical concern is to find a balance in the costs of procurement, production, distribution and storage against the customer service to be offered. Ben Naylor, Naim and Berry (1999) proposed a PC supply chain case study to discuss the decoupling point with considering the application of market knowledge. The mathematical model approaches use mathematical models or simulation to find an optimal position of the decoupling point. Gupta and Benjaafar (2004) developed models to compute the costs and benefits of delaying differentiation in series production systems when the order lead times are load dependent, and applied the queuing theory to seek the optimal position of the decoupling point. Viswanadham and Raghavan (2000) proposed a Petri nets model for decoupling point design and performed a simulation based on generalized stochastic Petri nets to minimize the sum of inventory carrying cost and the delayed delivery cost. 
Most of the mathematical model approaches assume that the decoupling point is the unique decision variable, or hinge on considering a static or steady state equilibrium of supply chains over an infinite planning horizon. However, the nature of the problem is dynamic and the planning horizon is finite. Today it is widely accepted that the product market undergo a life cycle of introduction, growth, maturity and eventual decline while accompanying with demand change. In recent years, some researchers discussed production-inventory models under demand uncertainty with time-varying targets by satisfying some certain state equation, we refer to the works by Emamverdi, Karimi \& Shafiee (2011), Baten \& Kamil (2009), Benhadid, Tadj and Bounkhel (2008), Choi, Reaff and Lee (2005) for detail. These indicate that the assumption of dynamic environment which is adjusted to the available demand information is more realistic in the realm of supply chain management.

In addition, many previous works on decoupling point are regardless of the deterioration of products. Deterioration is defined as decay, damage, spoilage, evaporation, loss of utility or loss of marginal value of commodity. The examples can be found in electronic components, fashion goods, medicine, fresh products and others. Since Ghare and Schrader (1963) firstly introduced the deteriorating items in inventory model and discussed the EOQ model with direct spoilage and exponential deterioration, the consideration of deteriorating items in inventory models have received a lot of attention (Sarkar, 2013; Wang, Lin \& Yu, 2011; Li, Lan \& Mawhinney, 2010). A manufacturer for deteriorating products has to produce more products than the market demand, which in turns influence the production planning and inventory management (Balkhi \& Benkherouf, 2004; Cheng \& Wang, 2009; Hsu, Wee \& Teng, 2007).

In this paper, we consider a production-inventory problem in conjunction with the decoupling point control such that we can take the customer demand into consideration when scheduling the operation plan. It worth to note that some realistic situations such as deterioration, timevarying demand as well as the finite life cycle are adopted in our dynamic model to simultaneously determine the optimal position of the decoupling point, optimal path of the production rate and the inventory level. The problem is explicitly solved with optimal control theory and the behaviors of optimal decoupling point with different parameters are analyzed. The remainder of this paper is organized as follows. In section 2, we define the notations and assumptions and proposed the dynamic model. In section 3, the optimal solution of the model is explored and the analytical solutions are obtained by applying optimization tools. Section 4 presents a practical application in electronic supply chain to address some specific and convincing values through mathematical and numerical analyses. We draw a conclusion in section 5 . 


\section{Problem Description and Basic Model}

Table 1 presents the notations and parameters used in this paper.

\begin{tabular}{|l|l|}
\hline Notation & Description \\
\hline$T$ & Decoupling point in supply chain \\
\hline$P_{0}$ & Estimated demand rate (i.e., production rate) during the pre-decoupling point \\
\hline$P(t)$ & Production rate at time $\boldsymbol{t}$ during the post-decoupling point \\
\hline$\hat{P}(t)$ & The target production rate at time $t$ during post-decoupling point \\
\hline$t_{f}$ & Length of product life cycle, also represent the planning horizon \\
\hline$D(t)$ & Demand rate at time $\boldsymbol{t}$ \\
\hline$I(t)$ & The inventory level at time $t$ during post-decoupling point \\
\hline$\hat{I}(t)$ & The target inventory level at time $t$ during post-decoupling point \\
\hline$\theta(t)$ & The deterioration rate occurs at time $\boldsymbol{t}$ \\
\hline$k$ & Constant cost per unit deviation from target production rate \\
\hline$h$ & Constant cost per unit deviation from target inventory level \\
\hline
\end{tabular}

Table 1. Model Notations

In addition, the following assumptions are made in this paper:

- The items are subject to deterioration and there is no repair or replacement of deteriorated items.

- The production rate is greater than the demand rate due to the deteriorating.

- The length of product life cycle (i.e. the decision horizon) is finite. Since in today's competitive environment, the product generally undergo a life cycle of introduction, growth, maturity and eventual decline.

- No shortage is allowed, so the production rate during the pre-decoupling point $P_{0}>\hat{P}(0)$ where $\hat{P}(0)$ is the target production rate at decoupling point.

- All the goal rates must satisfy the state equation.

Consider time $t$ over a finite product life cycle of a single product. At $t=0$, the production process begin with a production rate $P_{0}$, where $P_{0}$ is the estimated value of market demand rate. During the pre-decoupling point, the production rate start with $P(t)=P_{0}$ from the raw material procurement to the decoupling point when $t$ shift from $t=0$ to $t=T$. At the decoupling point, the true demand rate $D(t)$ is realized during the product life cycle from time $t=T$ to $T+T_{0}$. When the production during the pre-decoupling point is finished, the inventory level would be $I(L)=P_{0} T$ where $L$ is the throughput time of the supply chain. Without loss of 
generosity, we can shift the time axis so that $t=L$ becomes $t=0$. Then the problem transforms to the optimization during the planning horizon, $t \in\left[0, T_{0}\right]$ with the initial inventory condition $I(0)=P_{0} T$. It follows that the inventory level $I(t)$ evolves at each instant of time $t$ according to the state equation (also see as Emamverdi, et al., 2011; Jeong, 2011):

$$
\dot{I}(t)=-\theta(t) I(t)+P(t)-D(t), \quad I(0)=I_{0}=P_{0} T
$$

The objective of this paper is to provide appropriate production plan so that it can balance between the frequent change of the optimal control problem for the frequent change of production rate and the inventory holding cost for the given target production rate and the target inventory level with deterioration. The objective consists of two classes: minimizing inventory cost, and maintaining inventory level. This was also used by Porter and Taylor (1972) and Riddalls and Bennett (2001). The objective function can be written in the following quadratic form:

$$
J=\frac{1}{2} \int_{0}^{t_{f}}\left\{h[I(t)-\hat{I}(t)]^{2}+k[P(t)-\hat{P}(t)]^{2}\right\} d t
$$

where penalties $h>0$ and $k>0$ are incurred for the inventory level $I(t)$ and the production rate $P(t)$ deviation from target inventory level $\hat{I}(t)$ and target production rate $\hat{P}(t), h$ and $k$ are also called weight coefficients. The objective is to determine the optimal production rate $P(t)$ to ensure that the deviation from the target production rate $\hat{P}(t)$, i.e., $k[P(t)-\hat{P}(t)]^{2}$ as well as the deviation of the inventory level $I(t)$ from the target inventory level $\hat{I}(t)$, i.e., $h[I(t)-\hat{I}(t)]^{2}$ are both minimized. In this case, the maintaining of target inventory level is regarded as minimizing inventory cost via an indirect way, so that we can realize the goal to minimize the inventory cost (for example the inventory holding cost, deteriorating cost and backlog cost) despite the exact total inventory is very difficult to be calculated, this approach simplified the model and make sure the analytical solution is achievable.

\section{Optimal solution}

In this section, we use the Pontryagin's maximum principle (Pontryagin, 1987; Kamien \& Schwartz, 2012) of optimal control theory to derive the optimal time path of inventory level, production rate and optimal position of decoupling point for the proposed control problem. 


\subsection{Optimal inventory rate and optimal production rate}

Denote the Hamiltonian by

$$
H=\frac{1}{2} h[I-\hat{I}]^{2}+\frac{1}{2} k[P-\hat{P}]^{2}+\lambda[-\theta I+P-D],
$$

Where $\lambda$ is the adjoint variable. From the Pontryagin Maximum Principle, we have

$$
\dot{\lambda}=-h(I-\hat{I})+\theta \lambda, \quad \lambda\left(t_{f}\right)=0,
$$

and

$$
0=\frac{\partial H}{\partial P}=k(P-\hat{P})+\lambda
$$

Note that the goal pair $(\hat{I} ; \hat{P})$ must satisfy the state equation, to be feasible (Emamverdi et al., 2011; Baten \& Kamil, 2009; Benhadid et al., 2008), we get

$$
\dot{I}(t)=-\theta(t) \hat{I}(t)+\hat{P}(t)-D(t)
$$

From (5), we have $P=-\frac{1}{\mathrm{k}} \lambda+\hat{P}$, and so, $\dot{I}=-\theta I-\frac{1}{k} \lambda+\hat{P}-D$.

Finding the optimal solutions to minimize the objective (2) with condition (1) is equivalent to solving the following differential equations

$$
\left\{\begin{array}{l}
\dot{I}=-\theta I-\frac{1}{k} \lambda+\hat{P}-D, I(0)=I_{0}, \\
\dot{\lambda}=-h I+\theta \lambda+h \hat{I}, \lambda\left(t_{f}\right)=0 .
\end{array}\right.
$$

By the first equation in (7), we have

$$
\frac{\lambda}{k}=-\dot{I}-\theta I+\hat{P}-D
$$

Also, differentiating the first equation in (7), we get

$$
\ddot{I}=-\dot{\theta} I-\theta \dot{I}-\frac{1}{k} \dot{\lambda}+\dot{\hat{P}}-\dot{D}
$$

From (6), (7), (8) and (9), the optimal inventory rate (optimal path) is a solution of the following Riccati equation

$$
\ddot{I}+\left(\dot{\theta}-\theta^{2}-\frac{h}{k}\right) I=\theta(D-\hat{P})-\frac{h}{k} \hat{I}+\dot{\hat{P}}-\dot{D}, I(0)=I_{0}
$$


and the optimal production rate (optimal control) satisfies the following Riccati equation

$$
\frac{d^{2}(P-\hat{P})}{d t^{2}}=\left(\dot{\theta}+\theta^{2}+\frac{h}{k}\right)(P-\hat{P}),
$$

with

$$
P(0)=P_{0}, \quad \dot{P}(0)=\theta(0)\left(P_{0}-\hat{P}(0)\right)+\frac{h}{k}\left(I_{0}-\hat{I}(0)\right)+\dot{\hat{P}}(0)
$$

Lemma 1. For the optimal control problem that minimizes the objective $J$ of (2) under the condition (1) with time-varying deterioration rate $\theta(t)$, the optimal inventory rate $I$ satisfies the Riccati equation (10), and the optimal production rate $P$ is determined by the Riccati equations (11) with (12).

Theorem 1. For the optimal control problem that minimizes the objective $J$ of (2) under the condition (1) with constant deterioration rate, i.e., $\theta(t)=$ constant, the optimal production rate is

$$
P=k_{1} e^{\sqrt{a} t}+k_{\gamma} e^{-\sqrt{a} t}+\hat{P}
$$

and the optimal inventory rate is

$$
I=\hat{I}+\left(I_{0}-\hat{I}(0)\right) e^{-\theta t}+\frac{k_{1}}{\theta+\sqrt{a}}\left(e^{\sqrt{a} t}-e^{-\theta t}\right)+\frac{k_{2}}{\theta-\sqrt{a}}\left(e^{-\sqrt{a} t}-e^{-\theta t}\right)
$$

Where $a=\theta^{2}+\frac{h}{k}$, and

$$
\left\{\begin{array}{l}
k_{1}=\frac{1}{2}\left(1+\frac{\theta}{\sqrt{a}}\right)\left(P_{0}-\hat{P}(0)\right)+\frac{h}{2 k \sqrt{a}}\left(I_{0}-\hat{I}(0)\right), \\
k_{2}=\frac{1}{2}\left(1-\frac{\theta}{\sqrt{a}}\right)\left(P_{0}-\hat{P}(0)\right)-\frac{h}{2 k \sqrt{a}}\left(I_{0}-\hat{I}(0)\right) .
\end{array}\right.
$$

Proof. Let $a:=\theta^{2}+\frac{h}{k}$. Solving the equation (11) with constant deterioration rate $\theta$ and initial conditions (12), we have

$$
P=k_{1} e^{\sqrt{a} t}+k_{2} e^{-\sqrt{a} t}+\hat{P},
$$

Where $k_{1}$ and $k_{2}$ are determined by the following equations

$$
\left\{\begin{array}{l}
k_{1}+k_{2}=P_{0}-\hat{P}(0), \\
k_{1}-k_{2}=\frac{\theta}{\sqrt{a}}\left(P_{0}-\hat{P}(0)\right)+\frac{h}{k \sqrt{a}}\left(I_{0}-\hat{I}(0)\right) .
\end{array}\right.
$$


Solving the above equations, we can get (15).

From (1) and (6), we have

$$
\frac{d(I-\hat{I})}{d t}+\theta(I-\hat{I})=P-\hat{P}
$$

with

$$
I(0)=I_{0}
$$

and so,

$$
\begin{aligned}
& I-\hat{I}=\left(I_{0}-\hat{I}(0)\right) e^{-\theta t}+e^{-\theta t} \int_{0}^{t}(P-\hat{P}) e^{\theta \tau} d \tau \\
& =\left(I_{0}-\hat{I}(0)\right) e^{-\theta t}+\frac{k_{1}}{\theta+\sqrt{a}}\left(e^{\sqrt{a} t}-e^{-\theta t}\right)+\frac{k_{2}}{\theta-\sqrt{a}}\left(e^{-\sqrt{a} t}-e^{-\theta t}\right) .
\end{aligned}
$$

The proof is complete.

The optimal production rate and optimal inventory rate in the case with constant deterioration rate are illustrated by numerical simulation shown in the Figure 2, where the parameters are taken as $\hat{I}=60, I_{0}=85, \hat{P}=65, t_{f}=10, \theta=0.01, h=2, k=3$.
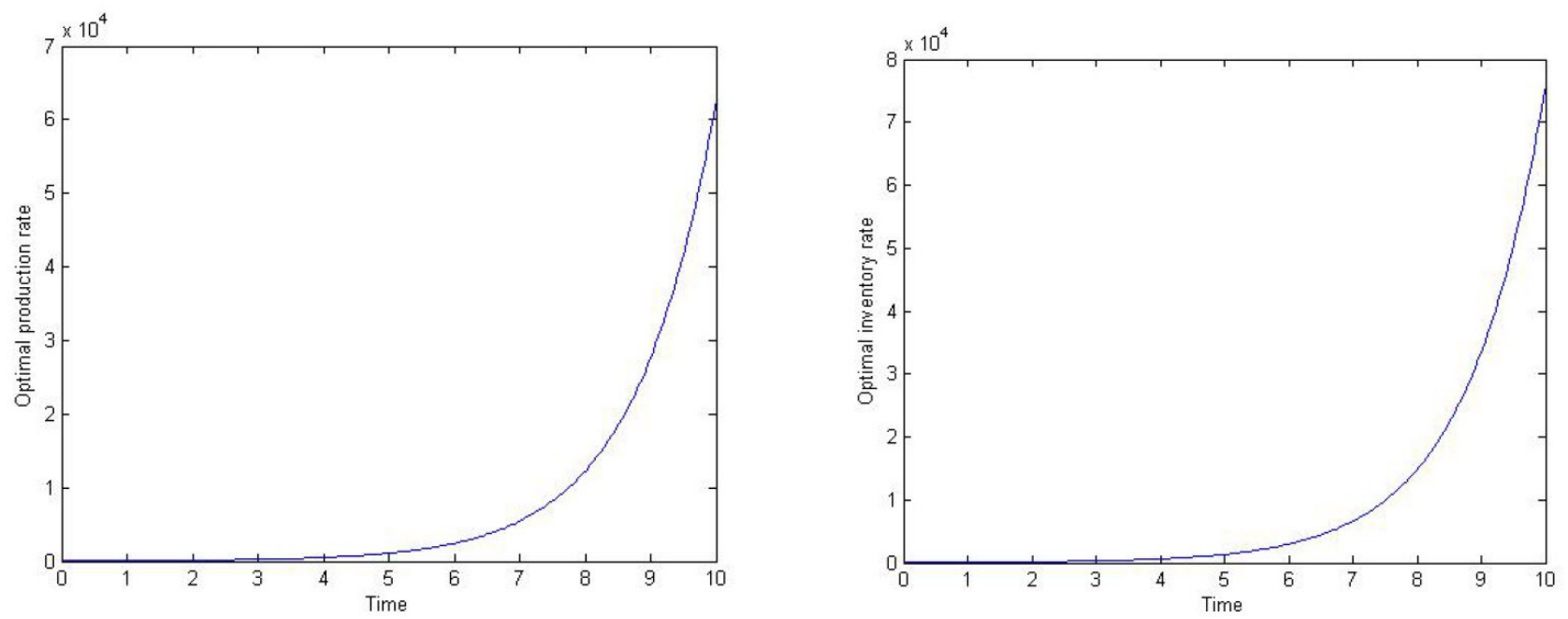

Figure 2. Optimal production rate and optimal inventory rate for parameters

$$
\hat{I}=60, I_{0}=85, \hat{P}=65, t_{f}=10, \theta=0.01, h=2, k=3
$$




\subsection{Optimal decoupling point}

From the initial condition in Equation (1),

$$
I_{0}=I(0)=P_{0} T
$$

Where $T$ is decoupling point in a supply chain. Equation (20) implies that the initial inventory level at the beginning of planning horizon is equal to the production quantity at the decoupling point $T$ with the estimated demand rate $P_{0}$.

Let $J=J(T)$ be the optimal objective function for the optimal control problem that minimizes the objective $J$ of (2) under the condition (1) with constant deterioration rate, then $J(T)$ can be differentiated as follows:

$$
\frac{\partial J}{\partial T}=\int_{0}^{t_{f}}\left\{h[I-\hat{I}] \frac{\partial I}{\partial T}+k[P-\hat{P}] \frac{\partial P}{\partial T}\right\} d t .
$$

Notice that

$$
\begin{gathered}
\frac{\partial I}{\partial T}=\frac{h P_{0}}{2 k \sqrt{a}}\left(\frac{e^{\sqrt{a} t}}{\theta+\sqrt{a}}-\frac{e^{\sqrt{a} t}}{\theta-\sqrt{a}}\right), \\
\frac{\partial P}{\partial T}=\frac{h P_{0}}{2 k \sqrt{a}}\left(e^{\sqrt{a} t}-e^{-\sqrt{a} t}\right) .
\end{gathered}
$$

Hence,

$$
\begin{aligned}
\frac{\partial J}{\partial T}= & \int_{0}^{t_{f}}\left[\frac{h^{2} P_{0}}{2 k \sqrt{a}}\left(\frac{k_{1}}{(\theta+\sqrt{a})^{2}}\right) e^{2 \sqrt{a} t}+\frac{k}{h}\left(k_{1}-k_{2}\right)-\frac{k_{2}}{(\theta-\sqrt{a})^{2}} e^{2 \sqrt{a} t}\right) \\
& \left.+\frac{h P_{0}}{2 \sqrt{a}}\left(k_{1} e^{2 \sqrt{a} t}-\left(k_{1}-k_{2}\right)-k_{2} e^{-2 \sqrt{a} t}\right)\right] d t \\
= & \int_{0}^{t_{f}}\left[e^{2 \sqrt{a} t} \frac{h P_{0} k_{1}}{2 \sqrt{a}}\left(\frac{h}{k(\theta+\sqrt{a})^{2}}+1\right)-e^{-2 \sqrt{a} t} \frac{h P_{0} k_{2}}{2 \sqrt{a}}\left(\frac{h}{k(\theta-\sqrt{a})^{2}}+1\right) d t\right) \\
= & \frac{h P_{0} k_{1}}{4 a}\left(\frac{h}{k(\theta+\sqrt{a})^{2}}+1\right)\left(e^{2 \sqrt{a} t_{f}}-1\right)+\frac{h P_{0} k_{2}}{4 a}\left(\frac{h}{k(\theta-\sqrt{a})^{2}}+1\right)\left(-e^{-2 \sqrt{a} t_{f}}-1\right),
\end{aligned}
$$

and

$$
\frac{\partial^{2} J}{\partial T^{2}}=\frac{h^{2} P_{0}}{9 k a \sqrt{a}}\left(\frac{h e^{2 \sqrt{a} t_{f}}}{k(\theta+\sqrt{a})^{2}}-\frac{h e^{-2 \sqrt{a} t_{f}}}{k(\theta-\sqrt{a})^{2}}+e^{2 \sqrt{a} t_{f}}-e^{-2 \sqrt{a} t_{f}}+\frac{4 k \theta \sqrt{a}}{h}\right)>0
$$

From the analyses, we can draw a conclusion as follows. 
Theorem 2. The optimal objective function is convex on decoupling point. There exists the unique optimal decoupling point expressed as: $T^{*}=\left(1-\frac{\hat{P}(0)}{P_{0}}\right) \frac{1-e^{2 \sqrt{a} t_{f}}}{(\sqrt{a}-\theta) e^{2 \sqrt{a} t_{f}}+(\sqrt{a}+\theta)}+\frac{\hat{I}(0)}{P_{0}}$.

\section{Discussion and application}

In real life scenario, many industries suffer from deteriorating items such as electronic component industry, fashion products, food and agricultural industry, to name a few. To further grip the implication of decoupling point on deterioration supply chains, the electronics supply chain dealing with the optimal control of decoupling point as well as the production-inventory plan is discussed in this section.

It is worthy to note that an electronics supply chain is a system of facilities that encompasses suppliers, manufacturers, transporters, retailers and customers. The suppliers give manufacturers their materials, semi-finished components and accessories through transporters by a large batch with a long lead time. The electronic manufacturers located in the middle echelon of the supply chain composes and produces final products and delivery them to retailers with small batches. Finally, the retailers sell the products to end customers. The supply chain members need to make responsive decisions on the position of optimal decoupling point, quantity of products, timing of production duration, and inventory level to guarantee the lowest cost as well as the flexibility of supply chain to commit the market share.

\subsection{Behavior of the deterioration rate}

The electronics industry is well known as a fertile field that enjoys huge profit along with considerable market demand. Unfortunately, the timely deterioration accompanied with rapid increasing in cost deeply confused the electronic enterprises. It forced them to take full consideration about deterioration in decision making. Therefore, we attack the behavior of the deteriorating rate on optimal decoupling point.

According to our model, the production planner estimates the demand rate $P_{0}$ during the predecoupling point and decides the target production rate $\hat{P}(0)$ at decoupling point, from the assumption of no shortage we have $P_{0}>\hat{P}(0)$. To take account of the deterioration rate on the position of optimal decoupling point, we have the following proposition. 
Proposition 1. The optimal decoupling point is monotonically decreasing with deterioration rate, the position of decoupling point shifts backwards to upstream suppliers with respect to deterioration rate increasing.

Proof. From Theorem2, we have

$$
\frac{\partial T^{*}}{\partial \theta}=\left(1-\frac{\hat{P}(0)}{P_{0}}\right) \frac{-(\sqrt{a}-\theta) e^{4 \sqrt{a} t_{f}}+2 \sqrt{a}\left(1-2 \theta t_{f}\right) e^{2 \sqrt{a} t_{f}}-(\sqrt{a}+\theta)}{\sqrt{a}\left[(\sqrt{a}-\theta) e^{2 \sqrt{a} t_{f}}+(\sqrt{a}+\theta)\right]^{2}}
$$

Notice that the $1-2 \theta t_{f}$ is non-negative if and only if $\theta \geq 0$ is enough small, and $\left[-(\sqrt{a}-\theta) e^{4 \sqrt{a} t_{f}}+2 \sqrt{a}\left(1-2 \theta t_{f}\right) e^{2 \sqrt{a} t_{f}}-(\sqrt{a}+\theta)\right]_{\theta=0}=0$. Therefore, $\frac{\partial T^{*}}{\partial \theta}<0$ when $P_{0}>\hat{P}(0)$. This completes the proof.

The relationship between the optimal decoupling point and deterioration rate is illustrated by the numerical simulation shown in Figure 3, where the parameters are taken as $h=2, k=3$ $\hat{I}=60, I_{0}=85, \hat{P}=65, t_{f}=10, \theta=0.01$.

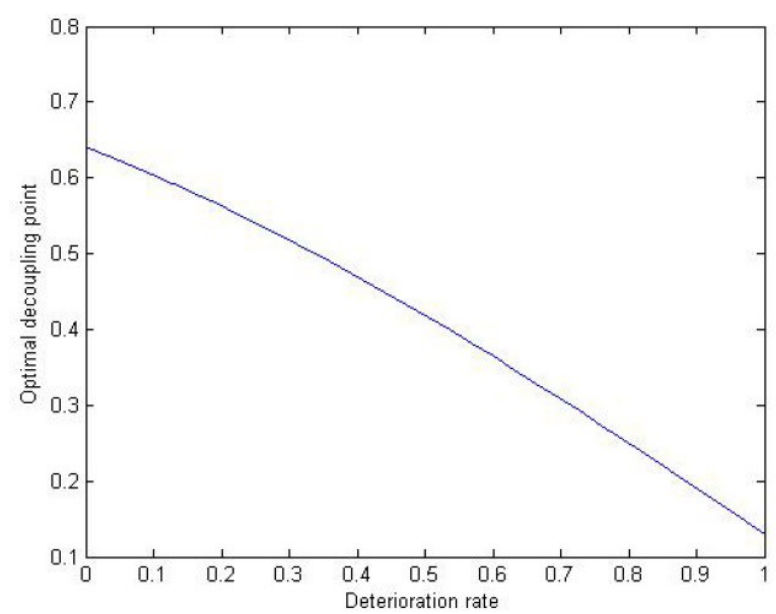

Figure 3. The relationships between the optimal decoupling point and deterioration rate where,

$$
\hat{I}=60, I_{0}=85, \hat{P}=65, t_{f}=10, \theta=0.01, h=2, k=3 .
$$

From the Proposition 1 we can imply that in order to minimize the inventory cost which is increased gradually with deterioration rate, the optimal decoupling point moves backwards to the upstream of supply chain as far as possible. It was demonstrated that with the purpose to maximal channel profit in practical, the optimal decoupling point of personal computer (PC) supply chains flowing closer to suppliers when compared with television (TV) supply chains since a higher deterioration rate happens to PC in common. By contrast, the length of maketo-stock of PC supply chains should be condensed to decrease the WIP (work in process). A 
production-inventory plan for PC supply chain would be hatched in view of fulfilling customer orders, following which an operational decision would be devised considering the reduction in inventory level and enhancement of customization.

\subsection{Effect of the length of product life cycle}

Since the modern business era is characterized by the frequently fluctuating in consumer demands, the tendency of rapid updating in generation and sustained shortening in product life cycle scattered over the electronic industry. Satisfying the variety demand of consumers and achieving shorter order-to-delivery times while allowing customers to customize their orders have played a significant role on an electronic firm's way to successful. Hence, we performed analysis about the length of product life cycle to examine the influence on optimal solution and practical decision.

In our model, a finite length of product life cycle (i.e. the finite planning horizon) is assumed for a more realistic setting. Additionally, we will also talk about the infinite horizon for comparison. The following proposition about product life cycle $t_{f}$ holds:

Proposition 2. The optimal decoupling point is monotonically an ascending function of the product life cycle $t_{f}$, the decreasing of product life cycle results in forward shifting to end customers. Anyway, if $t_{f} \rightarrow \infty$, the optimal decoupling point $T^{*}$ has a limit as $T^{*}=\left(1-\frac{\hat{P}(0)}{P_{0}}\right) \frac{-1}{(\sqrt{a}-\theta)}+\frac{\hat{I}(0)}{P_{0}}$.

Proof. From Theorem 2, we get

$$
\frac{\partial T^{*}}{\partial t_{f}}=\left.\left(1-\frac{\hat{P}(0)}{P_{0}}\right) \frac{-4 e^{4 \sqrt{a} t_{f}}\left[(\sqrt{a}-\theta) e^{2 \sqrt{a} t_{f}}+\theta\right]}{(a-\theta \sqrt{a}) e^{2 \sqrt{a} t_{f}}+(a+\theta \sqrt{a})}\right|^{2}
$$

Hence, $\frac{\partial T^{*}}{\partial t_{f}}<0$ when $P_{0}>\hat{P}(0)$.

Take $t_{f} \rightarrow \infty$, then $\lim T^{*}=\left(1-\frac{\hat{P}(0)}{P_{0}}\right) \frac{-1}{\sqrt{a}-\theta}+\frac{\hat{I}(0)}{P_{0}}$. These complete the proof. 
The relationship between the optimal decoupling point and final time is illustrated by the numerical simulation shown in Figure 4, where the parameters are taken as $h=2, k=3$ $\hat{I}=60, I_{0}=85, \hat{P}=65, t_{f}=10, \theta=0.01$,

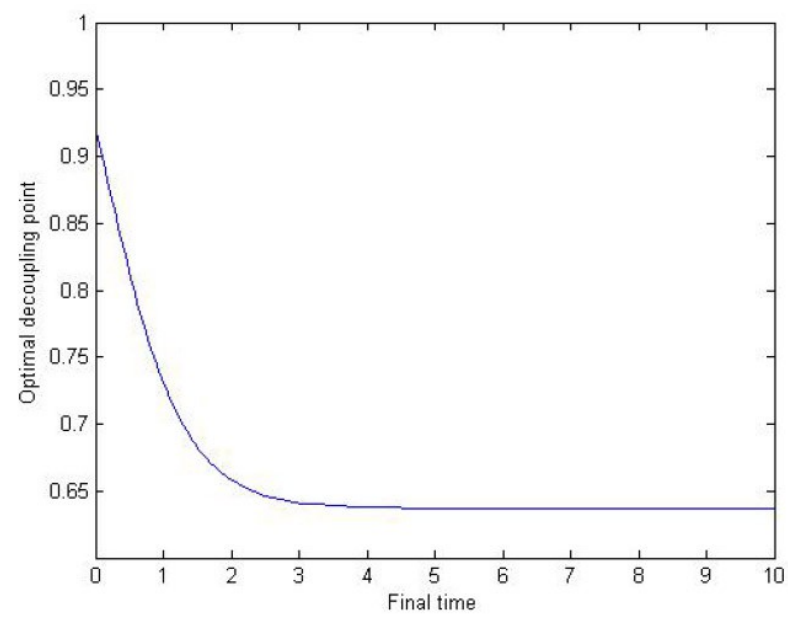

Figure 4. The relationships between the optimal decoupling point and final time where

$$
\hat{I}=60, I_{0}=85, \hat{P}=65, t_{f}=10, \theta=0.01, h=2, k=3 .
$$

Proposition 2 denote that under the consideration of infinite planning horizon that is $t_{f} \rightarrow \infty$, the optimal decoupling point is determined by the initial value while regardless of the change of target settings. The assumption of finite horizon conducts that the optimal decoupling point moves downstream to the end customers with the shortening of product life cycle. It can be implied that the optimal decoupling point of mobile phone supply chains is closer to end customers when compared to PC supply chains since the mobile phone market updating more frequently than computer. It denote that the there is more difficult for mobile phone manufactures to allow the customer customize their orders. This maybe one of the most important reasons that no mobile phone enterprise sells their product like Dell, a computer manufacture who is famous for its direct marketing which characterized by customization. However, the forward shifting of decoupling point can seize the initiative opportunity to expend the market share and satisfy the customer demand with quick response.

\section{Conclusion}

In this paper, we generalize and reformulate the model presented by In-Jae Jeong (2011). A combination of decoupling point and deterioration supply chains is put forward to explore a way for enterprises to achieve flexible and agile supply chain with deteriorating items while chasing for cost minimization. A dynamic model with deteriorating items was proposed to 
simultaneously determine the optimal position of decoupling point and production-inventory plan. By using the optimal control theory and analytical approach, we derived the closed form of the optimal decoupling point as well as the optimal time path of production rate and inventory level when the goal rates satisfy the state equation.

These results from mathematical and practical clearly reflect two major properties. The first one is that, under the deteriorating items, the optimal decoupling point is convex function on time and there exists the unique optimal decoupling point. The second one is that the optimal decoupling point changes is closely connected with the environments changes, such as deterioration rate and the length of the product life cycle. The position of optimal decoupling point shifts backwards to upstream suppliers with respect to deterioration rate increasing, while shifts forwards to end customers obviously with product life cycle decreasing. The logical manner of surveyed system characteristics can be considered as a validation for the proposed model and the performed computations.

Based on the findings presented herein, it is possible to extend the results of this research in several different directions. Further researches can spare effort to develop a tradeoff policy between different production policies. The inclusion of more realistic assumptions is necessary such as the time-varying penalty costs. Moreover, a consideration of delivery lead time to customer may enhance our understanding of the decoupling point phenomena.

\section{Acknowledgment}

This research is supported by the National Natural Science Foundation of China (No. 71272209), and Humanity and Social Science Foundation of Ministry of Education of China (No. 12YJA630170), and the National Natural Science Foundation of Hunan Province (NO. 12JJ3081), Universities Special Research Foundation of Central (No. 11HDSK306). The authors would like to give our great appreciates to all editors contribute to this research.

\section{References}

Agarwal, A., Shankar, R., \& Tiwari, M.K. (2006). Modeling the metrics of lean, agile and leagile supply chain: An ANP-based approach. European Journal of Operational Research, 173(1), 211-225. http://dx.doi.org/10.1016/j.ejor.2004.12.005

Balkhi, Z.T., \& Benkherouf, L. (2004). On an inventory model for deteriorating items with stock dependent and time-varying demand rates. Computers and Operations Research, 31(2), 223-240. http://dx.doi.org/10.1016/S0305-0548(02)00182-X 
Baten, A., \& Kamil, A.A. (2009). Analysis of inventory-production systems with Weibull distributed deterioration. International Journal of Physical Sciences, 4(11), 676-682. http://dx.doi.org/10.1016/j.ijps. ED8434F19782

Ben Naylor, J., Naim, M.M., \& Berry, D. (1999). Leagility: integrating the lean and agile manufacturing paradigms in the total supply chain. International Journal of production economics, 62(1), 107-118. http://dx.doi.org/10.1016/S0925-5273(98)00223-0

Benhadid, Y., Tadj, L., \& Bounkhel, M. (2008). Optimal control of production inventory systems with deteriorating items and dynamic costs. Applied Mathematics E-Notes, 8, 194-202. http://dx.doi.org/10.1016/j.amen.2008.07.031

Cheng, M., \& Wang, G. (2009). A note on the inventory model for deteriorating items with trapezoidal type demand rate. Computers \& Industrial Engineering, 56(4), 1296-1300. http://dx.doi.org/10.1016/j.cie.2008.07.020

Choi. J., Realff, M.J., \& Lee, J.H. (2005). Stochastic Dynamic Programming with Localized Costto-go Approximators: Application to large scale supply chain management under demand uncertainty. Chemical Engineering Research and Design, 83(6), 752-758.

http://dx.doi.org/10.1205/cherd.04375

Emamverdi, G.A., Karimi, M.S., \& Shafiee, M. (2011). Application of Optimal Control Theory to Adjust the Production Rate of Deteriorating Inventory System (Case Study: Dineh Iran Co.). Middle-East Journal of Scientific Research, 10(4), 526-531. http://dx.doi.org/10.1016/j.mesr.10.27.02

Ghare, P.M., \& Schrader, G.F. (1963). A model for exponentially decaying inventory. Journal of Industrial Engineering, 14(5), 238-243.

Gupta, D., \& Benjaafar, S. (2004). Make-to-order, make-to-stock, or delay product differentiation? A common framework for modeling and analysis. IIE transactions, 36(6), 529-546. http://dx.doi.org/10.1080/07408170490438519

Hoekstra, S., Romme, J., \& Argelo, S.M. (1992). Integral Logistic Structures: Developing Customer-Oriented Goods Flow. New York: McGraw-Hill Book Co Ltd.

Hsu, P.H., Wee, H.M., \& Teng, H.M. (2007). Optimal ordering decision for deteriorating items with expiration date and uncertain lead-time. Computers and Industrial Engineering, 52(4), 448-458. http://dx.doi.org/10.1016/j.cie.2007.02.002

Jeong, I.J. (2011). A dynamic model for the optimization of decoupling point and production planning in a supply chain. International Journal of Production Economics, 131(2), 561-567. http://dx.doi.org/10.1016/j.ijpe.2011.02.001

Kamien, M.I., \& Schwartz, N.L. (2012). Dynamic optimization: the calculus of variations and optimal control in economics and management. New York: Courier Dover Publications. 
Li, R., Lan, H., \& Mawhinney, J.R. (2010). A review on deteriorating inventory study. Journal of Service Science and Management, 3(1), 117. http://dx.doi.org/10.4236/jssm.2010.31015

Olhager, J. (2003). Strategic positioning of the order penetration point. International Journal of Production Economics, 85(3), 319-329. http://dx.doi.org/10.1016/S0925-5273(03)00119-1

Pontryagin, L.S. (1987). Mathematical Theory of Optimal Processes. Florida: CRC Press.

Porter, B., \& Taylor, F. (1972). Modal control of production-inventory systems. International Journal of Systems Science, 3(3), 325-331. http://dx.doi.org/10.1080/00207727208920270

Riddalls, C.E., \& Bennett, S. (2001). The optimal control of batched production and its effect on demand amplification. International Journal of Production Economics, 72(2), 159-168. http://dx.doi.org/10.1016/S0925-5273(00)00092-X

Sarkar, B. (2013). A production-inventory model with probabilistic deterioration in two-echelon supply chain management. Applied Mathematical Modeling, 37, 3138-3151.

http://dx.doi.org/10.1016/j.apm.2012.07.026

Soman, C.A., Van Donk, D.P., \& Gaalman, G. (2004). Combined make-to-order and make-to-stock in a food production system. International Journal of Production Economics, 90(2), 223-235. http://dx.doi.org/10.1016/S0925-5273(02)00376-6

Van Donk, D.P. (2001). Make to stock or make to order: The decoupling point in the food processing industries. International Journal of Production Economics, 69, 297-306. http://dx.doi.org/10.1016/S0925-5273(00)00035-9

Viswanadham, N., \& Raghavan, N.R.S. (2000). Performance analysis and design of supply chains: a Petri net approach. Journal of the Operational Research Society, 51, 1158-1169. http://dx.doi.org/10.2307/253928

Wang, K.J., Lin Y.S., \& Yu J.C.P. (2011). Optimizing inventory policy for products with timesensitive deteriorating rates in a multi-echelon supply chain. International Journal of Production Economics, 130, 66-76. http://dx.doi.org/10.1016/j.ijpe.2010.11.009

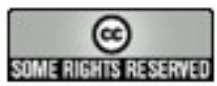

Article's contents are provided on a Attribution-Non Commercial 3.0 Creative commons license. Readers are allowed to copy, distribute and communicate article's contents, provided the author's and Journal of Industrial Engineering and Management's names are included. It must not be used for commercial purposes. To see the complete license contents, please visit http://creativecommons.org/licenses/by-nc/3.0/. 